\title{
Bogoliubov-De Gennes Formalism for Tracking Free Majoranas on Ultra Cold Fermi Gases
}

\section{Extensión del formalismo de Bogoliubov-De Gennes para el rastreo de fermiones de Majorana libres en gases fermionicos ultrafríos}

\author{
Angélica Alejandra Pérez Losada1,2, ${ }^{*}$, Karen Rodríguez Ramirez 1,2, Arturo Argüelles ${ }^{3}$ \\ 1. Departamento de Física, Universidad del Valle, A.A. 25360, Cali, Colombia \\ 2. Centro de Investigación e Innovación en Bioinformática y Fotónica - CIBioFi, Calle 13 No. 100-00, Edificio \\ 320, No. 1069, Universidad del Valle, Cali, Colombia \\ 3. Área de Matemáticas, Facultad de Ciencias Básicas, \\ Universidad Santiago de Cali, Calle 5 No. 62-00, Cali, Colombia \\ (") E-mail: angelica.perez@correounivalle.edu.co
}

Received: 18/12/2017 Accepted:21/08/2018

DOI: $10.7149 /$ OPA.51.3.50313

\begin{abstract}
:
The collective excitations of a system, particularly at low temperatures, behave as quasi-particles with properties that may differ from their constituents. In particular, there are exotic excitations called Majorana fermions or zero-energy modes modes, which have the special characteristic of being their own antiparticle. In that sense, this work seeks tracing these excitations in an artificial arrangement of a nanowire simulated through a one-dimensional chain. In that direction, the Fourier transform is used with the purpose of bringing the Hamiltonian from position to momentum space. Subsequently, it is proposed to diagonalize the system using the Bogoliubov-de Gennes formalism. In this way, we obtain the phase diagram displaying the set of parameters for which zero energy modes are stabilized.
\end{abstract}

Key words: Majorana fermions, dispersion relation, Bogoliubov-de Gennes Hamiltonian.

\section{RESUMEN:}

Las excitaciones colectivas de un sistema, en particular a bajas temperaturas, se comportan como cuasipartículas con propiedades que pueden diferir de las partículas constituyentes. En particular existen excitaciones exóticas llamadas fermiones de Majorana o modos de borde de energía cero, las cuales tienen la característica especial de ser su propia antipartícula y por tanto son modos de energía cero. En ese sentido, este trabajo busca rastrear dichas excitaciones en un arreglo artificial de un nanohilo, simulando éste a través de una cadena unidimensional de átomos fermionicos ultrafríos. En esa dirección, se usa la transformada de Fourier con el propósito de llevar el Hamiltoniano del sistema desde el espacio de posición al espacio de momento y posteriormente, diagonalizar el sistema desde el formalismo de Bogoliubov-de Gennes. De ésta manera, se consigue realizar el diagrama de transición de fase del sistema, donde se muestra el conjunto de parámetros para los cuales se estabilizan dichos modos de energía nula.

Palabras clave: Fermiones de Majorana, redes ópticas, Hamiltoniano de Bogoliubov-de Gennes.

\section{REFERENCES AND LINKS / REFERENCIAS Y ENLACES}

[1] R.P. Feynman, "Simulating physics with computers", Int.J.Theor.Phys, 21 p.467 (1982).

[2] Michael H. Freedman, et al., "Topological quantum computation”: Bull. Amer. Math. Soc. 40, p. 31-38 (2003).

[3] Keisuke Fujii, "Quantum Computation with Topological Codes: From Qubit to Topological FaultTolerance", Springer, ISBN 978-981-287-996-7, (2015). 
[4] Bloch Immanuel, et al., "Many-body physics with ultracold gases”, Rev. Mod. Phys. 80 p. 885-964 (2008).

[5] Bloch Immanuel, et al., "Quantum simulations with ultracold quantum gases". Nat Phys 8 p. 17452473 (2012).

[6] Adams C.S., et al., "Laser cooling and trapping of neutral atoms", Progress in Quantum Electronics 21, p. 1 - 79 (1997).

[7] Katori Hidetoshi, et al., "Magneto-Optical Trapping and Cooling of Strontium Atoms down to the Photon Recoil Temperature”, Phys. Rev. Lett. 82 p. 1116-1119 (1999).

[8] Brown Benjamin L., et al., "Coherent Control of Ultracold Molecule Dynamics in a Magneto-Optical Trap by Use of Chirped Femtosecond Laser Pulses", Phys. Rev. Lett. 96 p. 173002 (2006).

[9] Kitaev A Y, "Unpaired Majorana fermions in quantum wires”, Physics Uspekhi, 44 (2001).

[10] Rainis Diego, et al., "Majorana qubit decoherence by quasiparticle poisoning”, Phys. Rev. B 85 p. 174533 (2012).

[11] Wilczek F., "Majorana returns", Nature Physics 5, 614-518 (2009).

[12] Gennes, P. G. D, "Superconductivity of Metals and Alloys", Advanced Book Program, Perseus Books, (1999).

[13] Kim Yong-Jihn, "Pairing in the Bogoliubov-de Gennes Equations", International Journal of Modern Physics B 11 p. 06 (1997).

[14] LLC, MultiMedia. “Topology in Condensed Matter: Tying Quantum Knots”, (2016), https://www.edx.org/es/course/topology-condensed-matter-tying-quantum-delftx-topocmx-0

[15] Stoudenmire E. M, et al., "Interaction effects in topological superconducting wires supporting Majorana fermions". En: Phys. Rev. B 84 p. 014503 (2011).

[16] Mourik V., et al., "Signatures of Majorana Fermions in Hybrid Superconductor Semiconductor Nanowire Devices", Science 336 p. 1003-1007 (2012).

[17] Bardeen J, et al., “Theory of Superconductivity”, Phys. Rev. 108 p. 1175-1204 (1957).

[18] Iskin M., et al., "Topological superfluid phases of an atomic Fermi gas with in -and out-of-plane Zeeman fields and equal Rashba-Dresselhaus spin-orbit coupling", Phys. Rev. A 87 p. 063627(2013).

[19] Ptoj A., et al., "Yu-Shiba-Rusinov states of impurities in a triangular lattice of NbSe2 with spin-orbit coupling", Phys. Rev. B 96 p. 184425(2017).

\section{Introducción}

Actualmente uno de los objetivos científicos que cobra gran interés es la búsqueda de herramientas que conduzcan a la formulación coherente de lo que se conoce como un computador cuántico $[1,2]$. Su importancia radica en la consecución de un sistema físico capaz de almacenar información ilimitada, a partir de uno de los principios fundamentales de la Mecánica Cuántica conocido como Principio de Superposición. Hoy en día su formalismo se traza en términos de Qubits o Bits Cuánticos. Un Qubit es el acople de dos estados aislados, un ejemplo particular es el acople entre dos estados, uno llamado fundamental y el otro estado excitado de un determinado átomo; por consiguiente, representa una herramienta ideal para guardar información. La inconveniencia de este acople es el corto tiempo de coherencia entre dichos estados, ocasionando que la información no perdure en el tiempo [3]. Una alternativa para solucionar tal inconveniente emerge con el campo de los Átomos Ultra-fríos, pues al encontrarse los átomos a temperaturas del orden $10^{-6} \mathrm{~K}$, permite acoplar estados con la misma energía, tal que, se otorga un control único sobre un determinado sistema físico. Una de las grandes utilidades de ésta materia prima es la construcción de Simuladores Cuánticos [4, 5], son capaces de emular sistemas físicos de materia condensada. Una vez se obtienen los átomos ultra-fríos, se confinan a través de trampas Magneto-ópticas y láseres contra-propagantes capaces de emular los potenciales periódicos de una red cristalina [6-8]. En esta dirección, se pueden estudiar y caracterizar los estados de diferentes sistemas físicos altamente controlados desde distintos modelos teóricos, en particular el estudio a desarrollar en este trabajo propone la posibilidad de realizar un simulador cuántico de un nanoalambre superconductor a través de una cadena unidimensional de átomos ultrafríos, teniendo como punto de partida el esquema de la cadena de Kitaev [9]. Este modelo, a pesar de ser un modelo de juguete, pues carece de espín; posibilita el estudio de ciertas excitaciones de energía cero llamadas fermiones de Majorana (MFs), las cuales se caracterizan por ser su propia anti-partícula $\left(\hat{\gamma}=\hat{\gamma}^{\dagger}\right)$ y en consecuencia conforman el modelo perfecto para el estudio de estados 
tipo qubits cuánticos, puesto que, el acople entre dos parejas de dichas cuasi-partículas es altamente coherente a lo largo de todo el sistema, debido a que dichas cuasi-partículas tienen el mismo valor de energía y por tanto no se produce desexcitación en el Quibit [10]. Éstas excitaciones tipo Majorana fueron inicialmente propuestas por Ettore Majorana como un formalismo puramente teórico en el campo de altas energías [11]. A raíz de este modelo físico en particular, el presente trabajo consigue extender el formalismo matemático utilizado para describir el problema en el espacio de posición, hasta una descripción consistente en el espacio de momento, para un número arbitrario de sitios en una red unidimensional de átomos en el caso particular en el que $k=0 \mathrm{y} k=\pi$. Fundamentalmente, se demuestra como a partir del Hamiltoniano correspondiente del sistema en el espacio de momento se puede llevar al formalismo del método de Bogoliubov-de Gennes [12,13]. Este formalismo permite desacoplar los estados asociados a cada grado interno de libertad del sistema en el espacio de momento, tal que sea posible identificar bajo qué parámetros de energía el sistema presenta modos de cero energía o excitaciones tipo Majorana; conduciendo así a la construcción del gráfico de transición de fase topológica del sistema.

\section{Cadena de Kitaev}

El modelo más simple que describe un hilo superconductor, con electrones sin espín y que presenta fermiones de Majorana libres aislados en los bordes, es la cadena de Kitaev [9], cuyo Hamiltoniano está dado por:

$$
\widehat{H}=\sum_{i=1}^{N}\left(-t\left(\hat{c}_{i}^{\dagger} \hat{c}_{i+1}+\text { h.c. }\right)-\mu \hat{c}_{i}^{\dagger} \hat{c}_{i}+-\Delta\left(\hat{c}_{i} \hat{c}_{i+1}+\text { h.c. }\right)\right)
$$

Donde $\mu$ es el potencial químico, $t$ es la energía de salto, $\Delta$ es la intensidad del apareamiento tipo Cooper y $\hat{c}_{i}\left(\hat{c}_{i}^{\dagger}\right)$ el operador de destrucción (creación) de un fermión en el sitio $i$ - ésimo. Con la intensión de reescribir el Hamiltoniano Ec.(1) en términos de los operadores de Majorana, se introduce una transformación unitaria dividiendo un fermión en dos partes, su parte real e imaginaría. La Fig. [1] muestra un bosquejo donde cada operador fermiónico está dividido en dos operadores de Majorana, dicha transformación se escribe,

$$
\hat{c}_{i}=\frac{\hat{\gamma}_{i, A}+I \hat{\gamma}_{i, B}}{\sqrt{2}}, \quad \hat{c}_{i}^{\dagger}=\frac{\hat{\gamma}_{i, A}-I \hat{\gamma}_{i, B}}{\sqrt{2}},
$$

siendo $\hat{\gamma}_{i, \beta}$, los operadores de Majorana del tipo $\beta,(\beta=\{A, B\})$ en el sitio $i$-ésimo de la cadena e $I$ la unidad imaginaria. Bajo la transformación unitaria Ec. (2), el Hamiltoniano de la Ec.(1) toma la forma,

$$
\widehat{H}=\sum_{i=1}^{N}\left((\Delta-t) \hat{\gamma}_{i, A} \hat{\gamma}_{i+1, B}-\mu \hat{\gamma}_{i, A} \hat{\gamma}_{i, B}+(\Delta+t) \hat{\gamma}_{i, B} \hat{\gamma}_{i+1, A}\right)+0\left(\hat{\gamma}_{1, B} \hat{\gamma}_{L, A}\right)+0\left(\hat{\gamma}_{L, B} \hat{\gamma}_{1, A}\right)
$$

Si se considera el caso límite en que sólo la contribución del potencial químico está presente y por tanto $\Delta=$ $t=0$, el Hamiltoniano resultante es:

$$
\widehat{H}=\sum_{i=1}^{N}\left(-\mu \hat{\gamma}_{i, A} \hat{\gamma}_{i, B}\right)
$$

Éste se expresa a través de un eslabón directo entre los Majoranas $A$ y $B$ en el mismo sitio, ver Fig.(1) panel (a), situación que muestra el caso usual de los fermiones y por tanto se le denomina fase trivial.

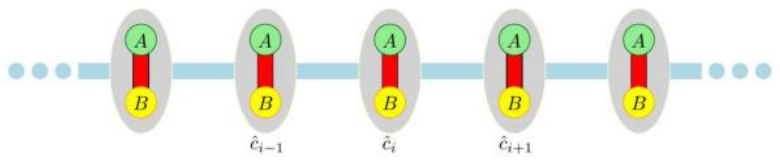

(a)

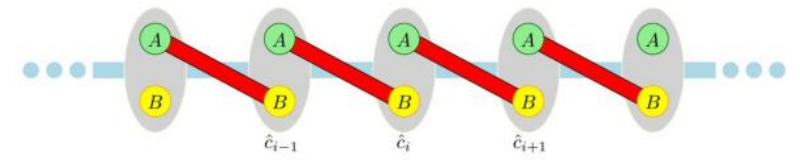

(b)

Fig.1: (a) Eslabón directo, fase trivial. (b) Eslabón indirecto, fase topológica, fermiones de Majorana libres en los bordes.

En el caso donde se anula el potencial químico $(\mu)$ y se igualan las contribuciones entre sitios vecinos, $\Delta=$ $t \neq 0$ el Hamiltoniano toma la forma:

$$
\widehat{H}=\sum_{i=1}^{N}\left((\Delta-t) \hat{\gamma}_{i, A} \hat{\gamma}_{i+1, B}+(\Delta+t) \hat{\gamma}_{i, B} \hat{\gamma}_{i+1, A}\right)
$$


y el acople energético entre la contribución debido a la formación de pares de Cooper y la energía de salto, origina un eslabón indirecto (enlace entre Majoranas $A$ y $B$ de sitios vecinos ver Fig. [1] panel (b)), en este caso a diferencia del anterior; se dice que el sistema se encuentra en una fase topológica. Debido a que la longitud de la cadena de átomos es finita y con fronteras fijas, se puede observar como un fermión se encuentra deslocalizado a lo largo de toda la cadena, resultado de los dos Majoranas libres en los extremos de la cadena que se acoplan, $\left(\hat{\gamma}_{L, B} \hat{\gamma}_{1, A}\right)$ o $\left(\hat{\gamma}_{1, B} \hat{\gamma}_{L, A}\right)$ apareciendo en el Hamiltoniano de la Ec. (3) con valor propio de energía cero, donde $L$ es el número de sitios de la red. Dicha superposición de una pareja de fermiones des localizados son identificados como un qubit o bit cuántico [2]. En general, si se levanta la restricción al potencial químico $(\mu=0)$ tal que, éste tome valores finitos, el par de fermiones de Majorana no están completamente localizados en los dos bordes del alambre, sino que decaen exponencialmente desde los bordes hacia el centro, tal que se estable una región trivial y una topológica, la cual se establece a medida que se rastrea la brecha de energía del sistema, mientras se cambia de forma simultánea el potencial químico y la energía de salto, como se observa en la Fig. [2]. En esta figura la energía está normalizada respecto a la energía de salto $(t)$, en ella se pueden identificar las dos fases que presenta el sistema y el conjunto de parámetros para los cuales se encuentra en una fase trivial, es decir, el espectro de energía presenta valores prohibidos de energía; el gap ( o brecha de energía) está abierto, región de color blanco, mientras que en la fase topológica el gap está cerrado, y por consiguiente existen estados de energía cero, región de color verde.

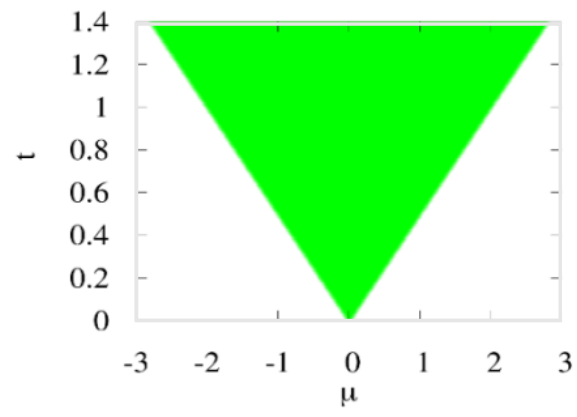

Fig. 2: Espectro de energía en el espacio de momentum normalizado respecto a la energía de salto para la cadena de Kitaev, La región verde corresponde a la fase topológica (gap cerrado), mientras que por fuera de esta región el sistema está en su fase trivial (gap abierto).

\section{2.a. Obtención de modos Majorana en superconductores unidimensionales}

La cadena de Kitaev constituye un modelo teórico adecuado para entender la fenomenología del los modos de energía cero. Sin embargo, al acercarse a un modelo más realista que permite describir sistemas de materia condensada, es necesario considerar el grado interno de libertad del sistema. Al agregar el espín, el esquema de una cadena unidimensional de átomos considerado hasta el momento se modifica. Ahora, se debe considerar la unión de dos cadenas de átomos, una ligada al espín $1 / 2$ y la otra ligada al espín $-1 / 2$, originando la formación del eslabón indirecto entre sitios vecinos para cada una de las cadenas, así, en los extremos de las cadenas se localizan fermiones de Majorana libres, dando lugar a la formación de dos fermiones complejos en los sitios extremos de las cadenas, eliminando la posibilidad de la formación de un fermion complejo a lo largo de toda la cadena , produciendo la perdida de los estados de borde, ya que no quedan fermiones de Majoranas libres (ver Fig.[3]).

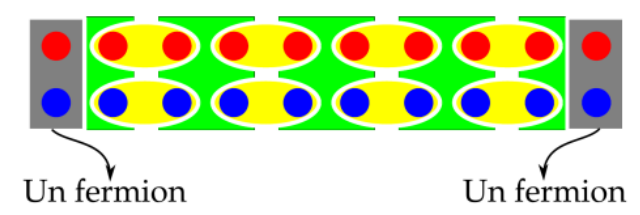

Fig. 3: Esquema cadena de Kitaev al introducir el grado interno de libertad. Se tienen dos cadenas asociadas a cada espín respectivamente, bolas rojas para espín y bola azules para espín. Nótese como el eslabón indirecto libera por sitio dos fermiones de Majorana y por tanto produce la perdida de los modos de borde en los extremos de las cadenas, cuando se encuentran dos fermiones de Majorana libres.

Para restablecer la fase topológica, aún bajo la adición del grado interno de libertad, es necesario la inclusión de un campo magnético en el sistema, el cual rompe la simetría SU(2) asociada a la inclusión del espín, de manera que separe las cadenas asociadas a cada proyección. Sin embargo, ésto no es suficiente para 
restitución de dichos modos. Por tanto, se debe incluir el acoplamiento espín-órbita $(\alpha)[18,19]$, el cual cumple el papel de eslabón indirecto nuevamente, pero en esta ocasión para fermiones de Majorana de distinto tipo entre sitios vecinos y espín opuesto. Para recuperar la fase topológica se debe romper la simetría de inversión temporal, que de acuerdo con la Ref. [15] solo se logra cuando se tienen las contribuciones energéticas descritas en la Ec.(6). El Hamiltoniano más completo para modelar el nanoalambre superconductor de la Fig. [4] es:

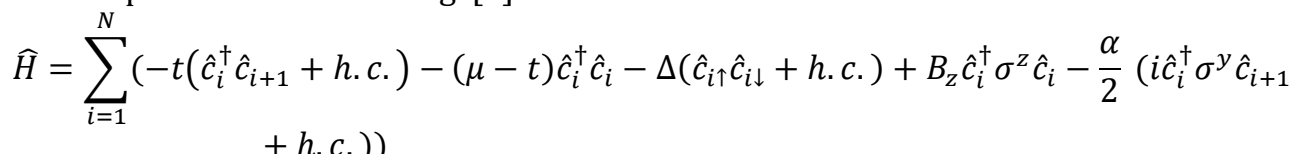

siendo la intensidad del campo magnético $B_{Z}$ la intensidad del acoplamiento espín-órbita es $\alpha$ y $\sigma^{z, y}$ las matrices de Pauli.

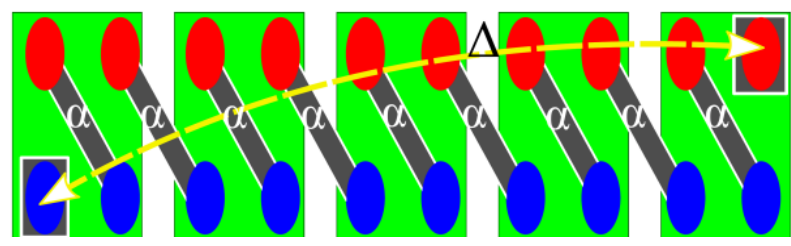

Fig. 4: Esquema de la cadena de Kitaev al incluir el espin (bolas rojas y azules para cada grado interno de libertad) y los acoples de $\mu, \mathrm{t}, \Delta, \mathrm{B}$ y $\alpha$, bajo la representación en fermiones de Majorana.

En este trabajo en particular se estudia uno de los arreglos artificiales que predice la formación de Majoranas en sistemas con espín, consta de disponer un nanohilo semiconductor en la proximidad de un superconductor, el nanohilo hereda un gap superconductor debido al apareamiento de pares de Cooper del superconductor, tal que al establecer el potencial químico para un valor entre el gap de energía, se produce la formación de modos de energía cero (fermiones de Majorana) en los extremos del nanohilo, y por tanto el gap del sistema se cierra [16]. Para entender por qué estos nanohilos no son superconductores normales sino superconductores topológicos, se debe recordar que el gap superconductor del sistema es una característica global, por lo tanto, constituye el parámetro de orden para determinar si el sistema está en una fase trivial o en una fase topológica. Ésto significa que, el sistema se encontrará en una fase trivial si bajo el cambio continuo de las contribuciones energéticas que intervienen en el sistema éste preserva su gap, y se establece la fase topológica si bajo dichos cambios continuos el gap se cierra.

\section{Transformación de Bogoliubov-de Gennes: Cadena de Kitaev}

Para obtener el diagrama de transición de fase de un nanohilo superconductor topológico, en el cual se delimitan las fronteras de transición de una fase trivial a una fase topológica y viceversa, se propone usar el método de Bogoliubov-de Gennes (BdG) $[12,13]$. Éste mecanismo es una generalización del modelo propuesto por Bogoluibov Valatin usado por Bardeen-Cooper-Schrieffer para describir su teoría de superconductividad [17]. El modelo funciona bajo la aproximación de campo medio, el cual permite reescribir el Hamiltoniano de cierto sistema físico, como contribuciones cuadráticas separadas multiplicadas por el campo de Ginzburg Landau $\Delta(x)=-g\left\langle\psi_{\uparrow}(x) \psi_{\downarrow}(x)\right\rangle$, siendo $g$ el parámetro de orden de dicha contribución. Posteriormente, buscando extender el estudio del sistema a un número arbitrario de sitios, se realiza una transformación unitaria que permita hacer el estudio del sistema en el espacio de momentum. En esta línea de ideas, una de las transformaciones que han sido bien estudiadas en este tipo de sistemas, es la transformación unitaria tipo Fourier para sistemas físicos que pueden considerarse periódicos infinitos; ésto significa que el tamaño o bulk del sistema es suficientemente grande en comparación a la longitud de coherencia entre los estados de las partículas, como es el caso de la teoría BCS. Sin embargo, en el caso particular del presente trabajo, se estudia un sistema finito y se debe establecer hasta qué punto trabajar con condiciones de frontera periódicas es conveniente para rastrear los modos de energía cero en el sistema. De manera que, el Hamiltoniano del sistema en espacio de momento se transforma en otra representación en términos del Hamiltoniano de Bogoliubov- de Gennes $\left(\widehat{H}_{B d G}\right)$, es decir, se reescribe el Hamiltoniano de la cadena de Kitaev matricialmente usando los llamados espinores de Nambu, como sigue $[12,19]$ :

$$
C^{\dagger}=\left(\begin{array}{ll}
\hat{c}_{k}^{\dagger}, & \hat{c}_{-k}
\end{array}\right), \quad C=\left(\begin{array}{c}
\hat{c}_{k} \\
\hat{c}_{-k}^{\dagger}
\end{array}\right)
$$


siendo $\hat{c}_{k}\left(\hat{c}_{k}^{\dagger}\right)$ el operador de destrucción (creación) con momento $k$, como resultado se obtiene una matriz Hamiltoniana efectiva conocida como $\widehat{H}_{B d G}$,

$$
\widehat{H}_{B d G}=\left(\begin{array}{cc}
\varepsilon_{k} & -\Delta_{k}^{*} \\
-\Delta_{k} & -\varepsilon_{k}
\end{array}\right)
$$

por lo tanto el Hamiltoniano del sistema toma la forma:

$$
\widehat{H}_{k}=\sum_{k} C^{\dagger} \widehat{H}_{B d G} C+\sum_{k} \varepsilon_{k} .
$$

El término independiente a la derecha de la Ec.(9), corresponde a un corrimiento en el espectro de energía del Hamiltoniano efectivo $\widehat{H}_{B d G}$. Este Hamiltoniano pese a ser una aproximación, proporciona un herramienta poderosa a la hora de construir el diagrama de transición de fase topológico del sistema, pues una vez se establecen correctamente los operadores de Nambu y se obtiene el $\widehat{H}_{B d G}$, al gráficar sus bandas en el espectro se evidencia un desdoblamiento en las mismas igual al número de grados internos de libertad del sistema. Como resultado de lo anterior se consigue rastrear en el espectro de $\widehat{H}_{B d G}$, bajo qué conjunto de parámetros la brecha de energía (gap) se cierra de manera que se identifiquen todos los modos de energía cero del sistema. En otras palabras, se rastrean el conjunto de parámetros para los cuales el sistema se encuentra en su fase trivial, es decir el gap está abierto y cuándo por el contrario está en su fase topológica, es decir el gap se cierra. El mecanismo anterior se aborda desde la representación en momento para condiciones de frontera periódicas (PBC). En este caso, se escribe el Hamiltoniano de la cadena de Kitaev, pasando directamente de la representación del Hamiltoniano en operadores fermionicos en posición al espacio de momentum. Una vez se tiene el Hamiltoniano en el espacio de momentum, se hace la transformación de BdG y se rastrea el gap para obtener el diagrama de transición de fase.

\section{3.a. Cadena de Kitev: condiciones de frontera periódicas (PBC)}

La transformación de BdG permite rastrear cuándo se cierra o abre el gap de energía de un cierto Hamiltoniano de acuerdo a lo descrito en la sección [1.a]. En el caso de estudio en cuestión, inicialmente se utiliza el Hamiltoniano de la cadena de Kitaev Ec.(1). Se usa la representación unitaria conocida como transformación de Fourier o expansión en ondas planas,

$$
\hat{c}_{i}=\frac{1}{\sqrt{N}} \sum_{k} e^{i k j} \hat{c}_{k},
$$

siendo $k$ el vector de onda y $N$ el número total de sitios de la cadena, tal que, el Hamiltoniano de la cadena de Kitaev transforma de la siguiente forma:

$$
\widehat{H}=\sum_{i=1}^{N}\left(\varepsilon_{k} \hat{c}_{k}^{\dagger} \hat{c}_{k}-\Delta_{k}^{*} \hat{c}_{k}^{\dagger} c_{-k}^{\dagger}-\Delta_{k} \hat{c}_{-k} \hat{c}_{k}\right),
$$

donde la relación de dispersión es $\varepsilon_{k}=-\mu-2 t \cos (k), \Delta_{k}^{*}=i \Delta_{k} \operatorname{con} \Delta_{k}=\Delta \sin (\mathrm{k})$, siendo $\Delta$ un número real. Una vez se tiene el Hamiltoniano escrito en el espacio de momentum Ec. (11), se procede a escribirlo en forma vectorial, para lo cual se usan los operadores de Nambu Ec.(7), como resultado se obtiene la matriz de acoples a la cual se le denomina Hamiltoniano efectivo de Bogoliubov-de Gennes $\left(\widehat{H}_{B d G}\right)$ Ec.(8), tal que el Hamiltoniano de estudio en términos de $\widehat{H}_{B d G}$, se escribe de la forma de la Ec.(9). Posteriormente se procede a rastrear el gap asociado de de los valores propios de $\widehat{H}_{B d G}$;

$$
\lambda_{1,2}= \pm \sqrt{\varepsilon_{k}^{2}+\Delta_{k}^{2}},
$$

Al graficar los valores propios de $\widehat{H}_{B d G}$ y al hacer la correspondiente diferencia entre los valores propios $\lambda_{1}$ y $\lambda_{2}$, tal que se rastreen el conjunto de parámetros para los cuales dicha diferencia de energía se hace igual a cero se obtiene la gráfica de la Fig. [5]. 


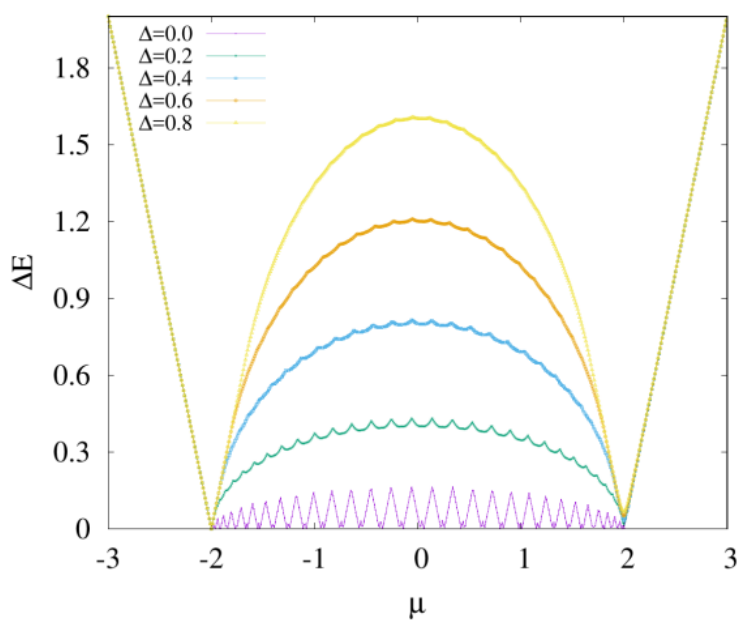

Fig. 5: Rastreo del gap de energía de para el caso en el que se asumen $\mathrm{PBC}$, siendo $t=1$. En este caso no hay dependencia con el número de sitios de la cadena.

En este gráfico se puede observar como el gap sólo se cierra completamente para los valores de $\mu=-2 \mathrm{y}$ $\mu=2$, mientras que para valores dentro de este intervalo se observan unas pequeñas oscilaciones que toman el valor de energía cero para determinados valores de $\mu$, ésto cuando $\Delta \ll 1$. Nótese que al incrementar $\Delta$ dicha oscilaciones se pierden y no se anula el gap. De acuerdo con la teoría el gap debe cerrase en la región en el que $\mu \epsilon[-2,2]$ (ver Fig.(2)). Por consiguiente al contrastar este resultado con el rastreo del gap en el espectro de energías de la cadena de Kitaev en el espacio de momentum, se observa cómo se favorece la formación de Majoranas en los extremos de la cadena para $\mu=0$ en la Fig. [2], mientras que para este mismo espectro en el espacio de momentum el gap no se cierra para este valor de potencial químico. En ese orden de ideas, se hace necesario esquematizar qué está ocurriendo con la representación en el espacio de momentum y analizar las implicaciones que conlleva usar la transformación unitaria tipo Fourier. Lo primero es pensar que al considerar condiciones de frontera periódicas la cadena de Kitaev deja de ser un sistema finito y luce como un anillo (ver Fig.[6.a]), tal que $\langle k \mid j=0\rangle=\langle k \mid j=L+1\rangle$, mientras que retomando la descripción discutida del Hamiltoniano de Kitaev en la representación de fermiones de Majorana (ver Ec.(3)) cuando $\mu=0$, se observa que los modos de energía cero se localizan en los extremos de la cadena, ésto debido a que se favorece la formación de eslabones indirectos en la cadena, dejando dos Majoranas libres en los extremos. Por lo tanto, al considerar PBC para $\mu=0$ se favorece la formación del eslabón indirecto y ésto en un anillo no admite modos de Majorana libres en los extremos de la cadena, ocasionando que no haya equivalencia entre las dos representaciones. Teniendo en cuenta que al haber quitado los fermiones de Majorana en los extremos usando PBC, sigue existiendo el gap para valores fuera del intervalo $\mu \epsilon[-2,2]$ del potencial químico, el gap asociado a estos valores es de tipo bloque, por lo tanto, para que se considere realmente la existencia de modos de energía cero asociados a los fermiones de Majorana, debe existir una correspondencia entre el bloque del sistema y los extremos, ésto en la literatura se conoce como correspondencia "Bulk-edge" [17].

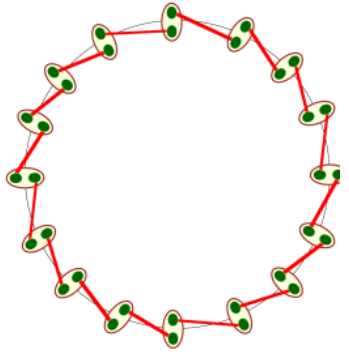

(a)

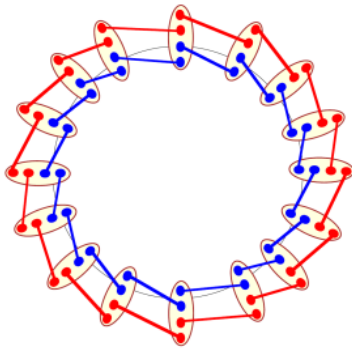

(b)

Fig. 6: (a) Esquematización cadena de Kitaev bajo PBC. Para $\mu=0$ se favorece la formación de un eslabón indirecto y no se producen fermiones de Majorana libres en los extremos de la cadena. (b): Representación esquemática de la cadena de Kitaev cuando se incluye el grado interno de libertad, bajo el uso de PBC. 


\section{3.b. Nano-hilo Superconductor: Condiciones de Frontera Periódicas (PBC)}

Con el propósito de entender e identificar lo que ocurre cuándo se consideran los diferentes tipos de condiciones de frontera e identificar hasta qué punto se puede validar o no el uso de PBC frente a los resultados previos en la Ref.[15] y los resultados del presente trabajo; se plantea desarrollar el modelo del nanohilo superconductor topológico en la representación de momento usando PBC. El Hamiltoniano más simple capaz de introducir todo el conjunto de contribuciones energéticas que intervienen en la construcción de un nanohilo superconductor topológico, es el Hamiltoniano de la Ec. (5). Este Hamiltoniano incluye a la cadena de Kitaev el grado interno de libertad, un campo magnético externo y el término asociado al acople espín-órbita. En la sección [2.a.] se discute el conjunto de acoples energéticos que se requieren para revelar los fermiones de Majorana libres en un arreglo experimental, de acuerdo con ésto, vale la pena esquematizar que le ocurre a la cadena de Kitaev cuando se incluye el grado interno de libertad (espín 1/2) y se consideran condiciones de frontera periódicas, bajo el conjunto de parámetros que favorecen la creación de eslabones indirectos entre Majoranas entre sitios vecinos $(\mu=$ $0, \Delta \neq 0$ y $t \neq 0$ ), se deberían evidenciar fermiones de Majoranas en los extremos de la cadena, sin embargo la formación de los eslabones entre sitios vecinos para cada grado de libertad en el anillo, anula la posibilidad de generar Majoranas libres (ver Fig.[6.b]). Entonces, de acuerdo con la descripción hecha en la sección [3.a], $k=0$ y $k=\pi$ son valores para los cuales la representación en PBC es acertada y constituye una buena aproximación del comportamiento del sistema, se procede a introducir el acoplamiento espínórbita y el campo magnético bajo el uso de la transformación usual de Fourier buscando identificar los límites de dicha representación. En la sección [1.a.] se explica como el Hamiltoniano de la Ec.(5) conduce a la formación de fermiones de Majorana. Adicionalmente se enuncia la existencia de una correspondencia entre el bloque y los extremos del sistema, tal que, cuando se rastrea el gap del sistema usando PBC, éste sólo representa un comportamiento coherente en los extremos de la primera zona de Brillouin. Así pues, pese a que el uso de PBC no describe el problema en su totalidad, conduce a resultados acertados para los valores de $k=0$ y $k=\pi$. Siguiendo este orden de ideas, al reescribir dicho Hamiltoniano en la representación de momento bajo $\mathrm{PBC}$, se obtiene:

$$
\begin{gathered}
\widehat{H}_{k}=\sum_{i=1}^{N}\left(\varepsilon_{k}\left(\hat{c}_{k \uparrow}^{\dagger} \hat{c}_{k \uparrow}+\hat{c}_{k \downarrow}^{\dagger} \hat{c}_{k \downarrow}\right)-\frac{\Delta}{2}\left(\hat{c}_{k \downarrow}^{\dagger} \hat{c}_{-k \uparrow}^{\dagger}-\hat{c}_{k \uparrow}^{\dagger} \hat{c}_{-k \downarrow}^{\dagger}+\hat{c}_{-k \uparrow} \hat{c}_{k \downarrow}-\hat{c}_{-k \downarrow} \hat{c}_{k \uparrow}\right)+B\left(\hat{c}_{k \uparrow}^{\dagger} \hat{c}_{k \uparrow}-\right.\right. \\
\left.\left.\hat{c}_{k \downarrow}^{\dagger} \hat{c}_{k \downarrow}\right)-\left(\alpha \hat{c}_{k \uparrow}^{\dagger} \hat{c}_{k \downarrow}+\alpha^{*} \hat{c}_{k \downarrow}^{\dagger} \hat{c}_{k \downarrow}\right)\right) .
\end{gathered}
$$

$\mathrm{Al}$ escoger los siguientes operadores de Nambu,

$$
C_{k}^{\dagger}=\left(\begin{array}{llll}
\hat{c}_{k \uparrow}^{\dagger}, & \hat{c}_{k \downarrow}^{\dagger}, & \hat{c}_{-k \uparrow}, & \hat{c}_{-k \downarrow}
\end{array}\right), \quad C_{k}=\left(\begin{array}{c}
\hat{c}_{k \uparrow} \\
\hat{c}_{k \downarrow} \\
\hat{c}_{-k \uparrow}^{\dagger} \\
\hat{c}_{-k \downarrow}^{\dagger}
\end{array}\right),
$$

el Hamiltoniano $H_{B d G}$ toma la forma:

$$
\widehat{H}_{B d G}=\left(\begin{array}{cccc}
\varepsilon_{k}+B & -\alpha_{k} & 0 & -\Delta \\
-\alpha_{k}^{*} & \varepsilon_{k}-B & \Delta & 0 \\
0 & \Delta & -\varepsilon_{k}-B & -\alpha_{k}^{*} \\
-\Delta & 0 & -\alpha_{k} & -\varepsilon_{k}+B
\end{array}\right)
$$

La relación de dispersión del $H_{B d G}$ haciendo $k=0$ o $k=\pi$ se muestra en la Fig.[6]. Al comparar estos resultados con resultados del gráfico de transición de fase obtenido en el trabajo previo de la Ref.[15], se observa cómo se ajustan perfectamente los resultados para $t=0$ (Fig.[6.b]). Nótese como al variar $t$ la región topológica se desplaza hacia la derecha si $t<0$, Fig.[6.a] y hacia la izquierda si $t>0$ Fig.[6.c], mientras que al variar el parámetro $\Delta$ dicha región se desplaza hacia arriba a medida que $\Delta$ aumenta positivamente. 


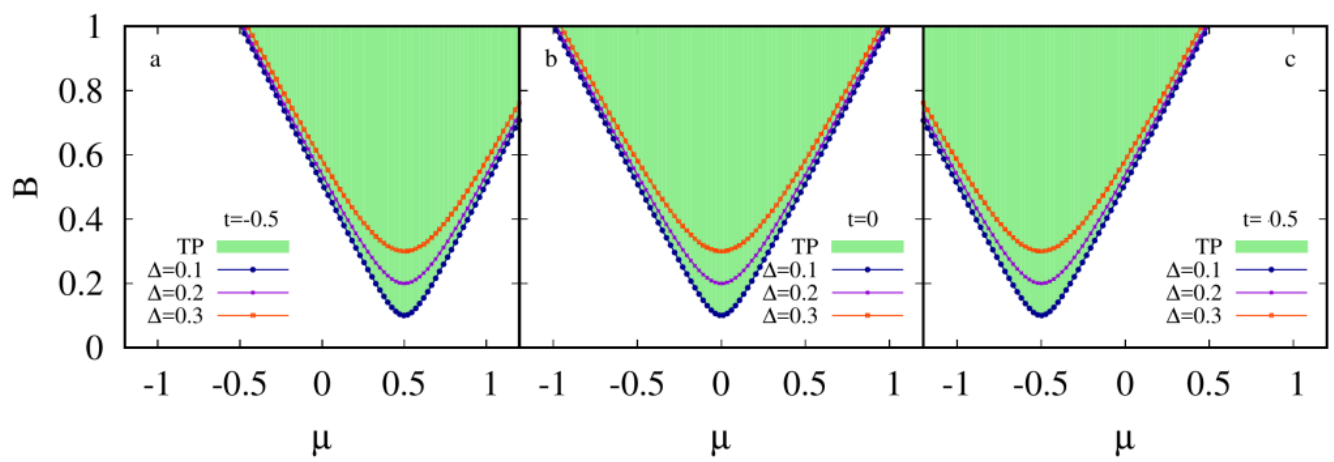

Fig. 6: Gráfico de transición de fase del para el caso en el que se asumen PBC tomando, para este caso se anula la dependencia con la contribución de acople espín-órbita. El conjunto de parámetros $(\mathrm{t}, \Delta, \mu$ y $B$ ) para los cuales el sistema está en su fase topológica corresponde a todos los valores en el interior de la curva, mientras que la región blanca corresponde a la región trivial.

De acuerdo con el resultado obtenido se puede observar como bajo PBC es posible tener una primera aproximación de la región topológica del sistema de estudio, sin embargo, ésto es sólo funciona para valores particulares de $k$. En este orden de ideas, estudiar qué ocurre con el gráfico de transición de fase cuando se incluyen las contribuciones de $B$ y $\alpha$ cuando se trabaja bajo condiciones de frontera fijas se planea desarrollar como una perspectiva al presente trabajo, donde se use una representación en el espacio de momento que proteja las condiciones de frontera fijas del sistema.

\section{Conclusiones}

Se identificaron las discrepancias y consistencias del modelo bajo el uso de PBC, teniendo en cuenta que los resultados obtenidos a partir del $\widehat{H}_{B d G}$ en la representación de momentum bajo PBC, se encuentra que dicha representación se ajusta perfectamente a los resultados obtenidos en trabajos posteriores en la representación en el espacio de posición (Ref.[15]) para el caso en el que el vector toma los valores de $k=0$ y $k=\pi$. Éste resultado permite trazar los límites bajo el uso de PBC y establecer como se modifica el gráfico de transición de fase del sistema cuando se modifican los parámetros $t, \Delta, \mu$ y $B$, siendo $\alpha$ arbitrario. Sin embargo, de acuerdo con trabajos posteriores la dependencia con $\alpha$ es fundamental y modifica el gráfico de transición de fase para valores arbitrarios de $k$. Por lo tanto debe trabajarse el problema bajo el uso de condiciones de frontera fijas, de manera que se proteja la geometría del sistema en la representación de momento.

\section{Agradecimientos}

Los autores agradecen al Centro de Investigación en Bioinformática y Fotónica - CiBioFi y al Sistema General de Regalías para la Ciencia, Tecnología e Innovación (Fondo CTeI-SGR) bajo el contrato No. BPIN 2013000100007 por el respaldo económico. 